\title{
Physics Design Considerations for Diagnostic X Electron Beam Transport System
}

Y-J. Chen

April 10, 2000

U.S. Department of Energy

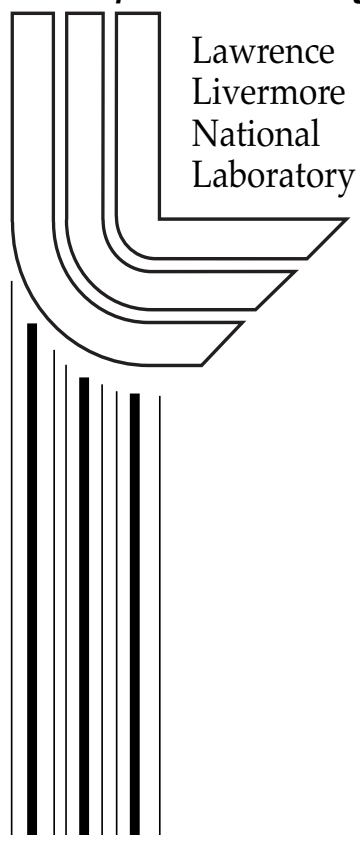




\section{DISCLAIMER}

This document was prepared as an account of work sponsored by an agency of the United States Government. Neither the United States Government nor the University of California nor any of their employees, makes any warranty, express or implied, or assumes any legal liability or responsibility for the accuracy, completeness, or usefulness of any information, apparatus, product, or process disclosed, or represents that its use would not infringe privately owned rights. Reference herein to any specific commercial product, process, or service by trade name, trademark, manufacturer, or otherwise, does not necessarily constitute or imply its endorsement, recommendation, or favoring by the United States Government or the University of California. The views and opinions of authors expressed herein do not necessarily state or reflect those of the United States Government or the University of California, and shall not be used for advertising or product endorsement purposes.

This work was performed under the auspices of the U. S. Department of Energy by the University of California, Lawrence Livermore National Laboratory under Contract No. W-7405-Eng-48.

This report has been reproduced directly from the best available copy.

Available electronically at http://www.doc.gov/bridge

Available for a processing fee to U.S. Department of Energy

And its contractors in paper from

U.S. Department of Energy

Office of Scientific and Technical Information

P.O. Box 62

Oak Ridge, TN 37831-0062

Telephone: (865) 576-8401

Facsimile: (865) 576-5728

E-mail: reports@adonis.osti.gov

Available for the sale to the public from

U.S. Department of Commerce

National Technical Information Service

5285 Port Royal Road

Springfield, VA 22161

Telephone: (800) 553-6847

Facsimile: (703) 605-6900

E-mail: orders@ntis.fedworld.gov

Online ordering: http://www.ntis.gov/ordering.htm

OR

Lawrence Livermore National Laboratory

Technical Information Department's Digital Library

http:/ / www.llnl.gov/tid/Library.html 


\title{
Physics Design Considerations for Diagnostic X Electron Beam Transport System
}

\author{
Yu-Jiuan Chen \\ April 10, 2000
}

The Diagnostic X (D-X) beamlines will transport the DARHT-II ${ }^{1}$ beam from the end of the accelerator to the Diagnostic $X$ firing point providing four lines of sight for $\mathrm{X}$ ray radiography. The design goal for the Diagnostic $\mathrm{X}$ beamline is to deliver four $\mathrm{X}$-ray pulses with the DARHT-II dose format and time integrated spot size on each line of sight. The D-X beamline's final focus should be compatible with a range of first conjugates from $1 \mathrm{~m}-5 \mathrm{~m}$. Furthermore, the D-X beamline operational parameters and the beamline layout should not preclude a possible upgrade to additional lines of sight. The DARHT-II accelerator is designed to deliver beams at a rate of 1 pulse per minute or less. Tuning the D-X beamline with several hundred optical elements would be time consuming. Therefore, minimizing the required number of tuning shots for the $\mathrm{D}-\mathrm{X}$ beamline is also an important design goal. Many different beamline configurations may be able to accomplish these design objectives, and high beam quality (i.e., high current and low emittance) must be maintained throughout the chosen beamline configuration in order to achieve the DARHT-II x-ray dose format. In general, the longer the distance a beam travels, the harder it is to preserve the beam quality. Therefore, from the point of view of maintaining beam quality, it is highly desirable to minimize the beamline length. Lastly, modification to the DARHT-II building and the downstream transport should be minimized.

Several processes can degrade beam quality by increasing the beam emittance, increasing the time-varying transverse beam motion, creating a beam halo, or creating a time-varying beam envelope. In this report, we consider those processes in the passive magnet lattice beamline and indicate how they constrain the beamline design. The physics design considerations for the active components such as the kicker system will be discussed in Ref. 2. In Sec. I, we discuss how beam emittance affects the X-ray forward dose. We also establish a physics design goal for the emittance growth budget. In Sec. II, we discuss how the conductivity and size of the beam pipe affects the transverse beam motion. We also discuss the emittance growth arise from the beam centroid offset. In Sec. III, we discuss the background gas focusing effects and establish the vacuum requirements. In Sec. IV, we consider the emittance growth in a bend. In Sec. V, we discuss the misalignment and corkscrew motion. The design specifications for misalignment are established. In Secs. VI and VII, we discuss the design objectives on how to extract beams from the DARHT-II beamline and how to minimize the tuning shots. The integrated spot size and final focusing are discussed in Sec. VIII. A conclusion will be presented in Sec. IX.

\section{Emittance and X-ray Dose}

The D-X transport system is to deliver four X-ray pulses with the DARHT-II X-ray dose format and time integrated spot size on each line of sight. Each D-X line of sight's 
beam path length is approximately $120 \mathrm{~m}^{3}$, which is about 10 times the DARHT-II downstream beamline length. While the beam on the DARHT-II beamline needs to transport through only one kicker system, the current pulse on a typical D-X beamline would travel through several large angle achromatic bend systems, one active D-X kicker system, several passive D-X kickers in addition to an active DARHT-II kicker system which chops beam in shorter pulses and dumps unwanted charge. One of the design goals for the DARHT-II transport and final focus system is to obtain a beam emittance no greater than $1500 \pi \mathrm{mm}$-mrad on the target if the exiting beam from the DARHT-II accelerator has an emittance no greater than $1000 \pi \mathrm{mm}$-mrad. Before we set the design goal for the final beam emittance on the Diagnostic X, we should examine how the X-ray dose varies with emittance.

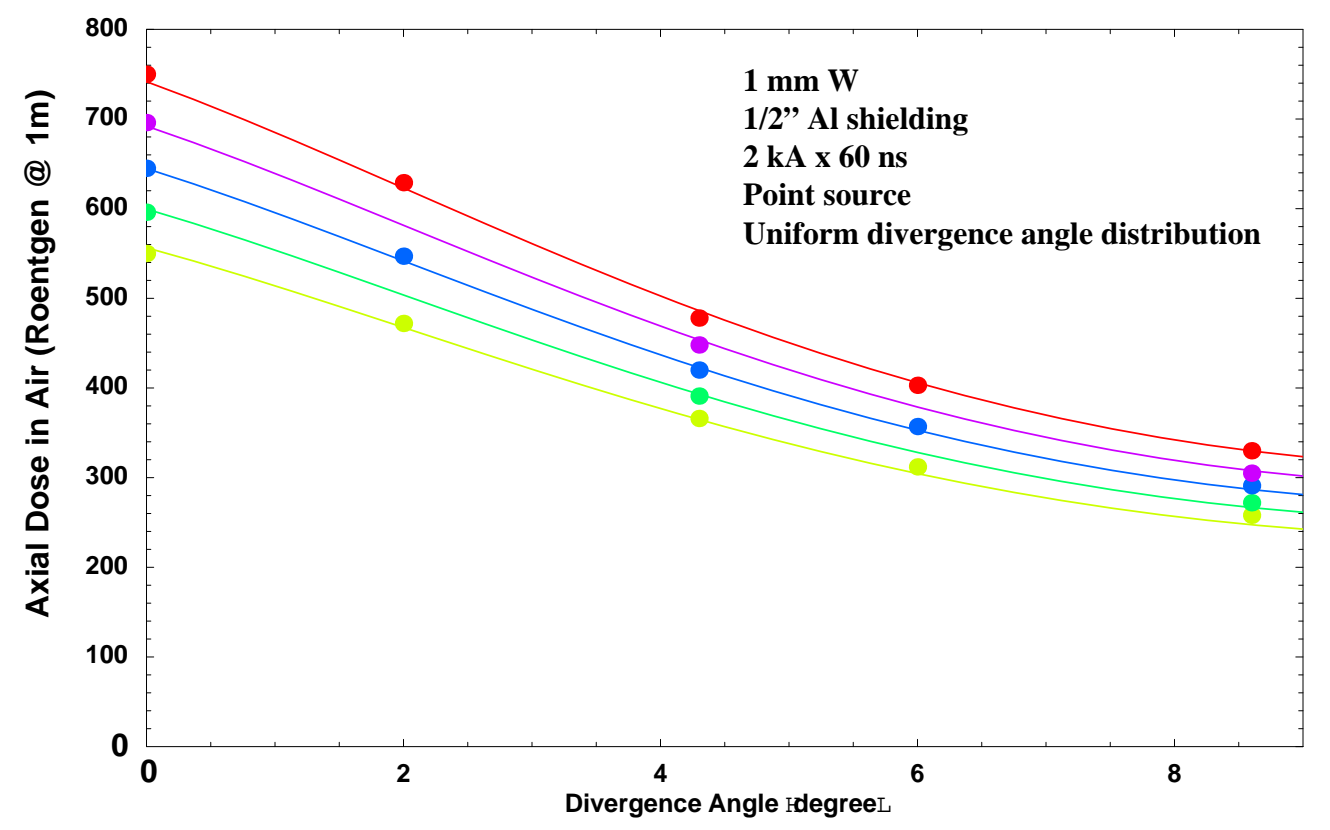

Fig. 1 The total, forward $\mathrm{x}$-ray dose in the air as a function of the beam energy and divergence angle of a $2 \mathrm{kA}, 60 \mathrm{~ns}$ long beam. An $1 \mathrm{~mm}$ thick tungsten is used as the $\mathrm{x}$-ray converter target. The axial $\mathrm{x}$-ray dose in the air calculations include the $\mathrm{x}$-ray attenuation in a $1 / 2$ inch thick aluminum plate. The beam energies for the curves from the top to the bottom are $20,19.5,19,18.5,18 \mathrm{MeV}$, respectively.

The Bremsstrahlung radiation from a relativistic electron beam is confined within a cone angle, which is approximately $1 / \gamma$. The internal divergence angle or thermal spread of the beam will increase the radiation cone angle, and hence lower the forward x-ray dose. Figure 1 shows how the beam divergence angle and beam energy affect the x-ray dose on the axis ${ }^{4}$. The marks indicate the calculation data points. The calculations assume that the electrons are focused to a singular point on a 1-mm thick tungsten plate. The total charge hitting the converter is $2 \mathrm{kA} \times 60 \mathrm{~ns}$. A measured $\mathrm{x}$-ray dose is a function of detector material. The calculations are done for the total doses (all photon energies) measured in the air for five beam energies; $18 \mathrm{MeV}, 18.5 \mathrm{MeV}, 19 \mathrm{MeV}, 19.5 \mathrm{MeV}$ and $20 \mathrm{MeV}$ (data shown from the bottom to the top). Fits with the functional form of 


$$
D \cong 2 \times 10^{-4} I[k A] \tau_{p}[n s] \gamma^{2.8} \exp \left[-0.07 \theta-0.0103 \theta^{2}+0.00087 \theta^{3}\right]
$$

are also shown, where $\tau_{p}$ is the current pulse length, $\gamma$ is the Lorentz factor, $\theta$ is the intrinsic beam divergence angle in degrees, and the unit for the dose $D$ is Roentgen at 1 $\mathrm{m}$. The conversion factor to rad is $1 \mathrm{R}=0.88 \mathrm{rad}$.

If there is a residual final focusing magnetic field on the target, the effective beam emittance is given as

$$
\varepsilon=\sqrt{\varepsilon_{o}^{2}+\omega_{c}^{2} a_{f}^{2}}
$$

where $a_{f}$ and $\omega_{\mathrm{c}}$ is the beam radius and the cyclotron angular frequency on the taregt, respectively, and $\varepsilon_{o}$ is the Lapostolle emittance which is also the edge emittance if the beam is uniformly distributed both in the physical space and the velocity space. If there is a $1 \mathrm{kG}$ magnetic field threading the DARHT-II target, the effective emittance is $1534 \pi$ mm-mr. From Eq. (1) and $\theta=\varepsilon / a_{f}$, we obtain the scaling law for the dose change in terms of the emittance change and the final spot size change as

$$
\frac{\Delta D}{D} \approx-\left[4.01+67.6\left(\frac{\varepsilon}{a_{f}}\right)-491\left(\frac{\varepsilon}{a_{f}}\right)^{2}\right]\left(\frac{\varepsilon}{a_{f}}\right)\left(\frac{\varepsilon_{o}^{2}}{\varepsilon^{2}} \frac{\Delta \varepsilon_{o}}{\varepsilon_{o}}-\frac{\Delta a_{f}}{a_{f}}\right),
$$

where $\varepsilon$ is the un-normalized emittance in cm-rad, and $a_{f}$ is the beam radius on the converter target in $\mathrm{cm}$. Note that the value in the square bracket is always positive. The intrinsic beam divergent angle increases when the emittance is larger or when the spot size is smaller, and hence, the forward x-ray dose decreases. With the DARHT-II target's specifications [2.1 mm spot size (diameter), $20 \mathrm{MeV}$ and $1500 \pi \mathrm{mm}-\mathrm{mr}$ of normalized emittance], we have

$$
\frac{\Delta D}{D} \approx-20 \%\left(\frac{\varepsilon_{o}^{2}}{\varepsilon^{2}} \frac{\Delta \varepsilon_{o}}{\varepsilon_{o}}-\frac{\Delta a_{f}}{a_{f}}\right) .
$$

The design goal for the D-X transport is to deliver the DARHT-II-like $\mathrm{x}$-ray dose pulse format to the test object. However, instead of accepting the DARHT-II emittance specification, let us assume that a $5 \%$ reduction in the x-ray dose is acceptable. Then, assuming that there is no magnetic field on the target leads to the design goal for the maximum acceptable beam emittance at the $\mathrm{D}-\mathrm{X}$ targets to be less than $1875 \pi \mathrm{mm}-\mathrm{mr}$.

We have now established the emittance growth budget for the beam traveling about $120 \mathrm{~m}$ from the DARHT-II accelerator exit to the D-X firing point as $875 \pi \mathrm{mm}$ mr. Comparing that with the emittance growth budget on the DARHT-II downstream transport of $500 \pi \mathrm{mm}-\mathrm{mr}$ for about $12 \mathrm{~m}$ indicates that it is extremely important and challenging to preserve the beam emittance in the $\mathrm{D}-\mathrm{X}$ beamline, for example minimizing the nonlinear external fields and the nonlinear image forces (or small beam and small 
offset), minimizing all possible instabilities, etc. The design magnetic tune for the D-X transport should be far away from any known parametric instabilities. The beam envelope in the D-X beamline and the beam spot size on a target should not be sensitive to the beam parameters and magnetic tune.

\section{Conductivity and Pipe Size}

Before one can layout a beamline transport lattice, the beampipe sizes for various transport systems need to be determined. There are several transport issues regarding choice of beam pipe size and material, especially, conductivity of the pipe material. The finite conductivity of the pipe wall can cause both transverse ${ }^{5}$ and longitudinal ${ }^{6}$ resistive $^{2}$ wall instabilities and beam energy loss.

\section{II.1 Transverse Resistive Wall Instability}

The transverse resistive wall instability arises from the diffusion of the return current into the beampipe wall due to its finite conductivity. The reduction in the magnetic forces of the dissipating return current acts as frictional forces on the particles. The difference in the frictional forces felt by the beam head and by the beam tail increases with the beam pulse length. While a beam traveling through a cylindrical beam pipe with a perturbation in the form of betatron oscillations, two waves (fast and slow) can be excited. The friction forces of the resistive wall damp the fast wave. On the other hand, the friction forces enhance the slow wave. For a slow wave, the particle motions are opposite to the beam centroid motion. Therefore, the friction forces actually accelerate and amplify particles betatron motion. The net effects of the friction forces on the slow wave of the transverse beam displacement would be accumulated over the length of the beam path and lead to beam loss and large time integrated spot sizes on the targets.

For a continuous focusing system, the transverse resistive wall instability's amplitude grows approximately as $\exp \left[1.5\left(z / L_{t r}\right)^{2 / 3}\right]$, where $L_{t r}$ is the instability's characteristic growth length $^{3}$ given as

$$
\begin{aligned}
L_{t r} & =\frac{2 \gamma \beta I_{o}}{I} \sqrt{\frac{\pi \sigma}{\tau} \frac{k_{\beta} b^{3}}{c}} \\
& =\frac{4 \gamma}{I[\mathrm{kA}]} \sqrt{\frac{\sigma\left[\mathrm{sec}^{-1}\right]}{10^{17} \tau[\mu \mathrm{s}]}}\left(\frac{b[\mathrm{~cm}]}{10}\right)^{3} \frac{100}{\lambda_{\beta}[\mathrm{m}]}[\mathrm{m}],
\end{aligned}
$$

where $k_{\beta}$ and $\lambda_{\beta}$ is the space charge suppressed betatron wavenumber and wavelength, respectively, $I$ is the beam current, $\gamma$ is the beam's Lorentz factor, $I_{o}$ is the Aflvén current $(\sim 17 \mathrm{kA}), \sigma$ and $b$ is the beampipe's conductivity and radius. For an emittance dominated beam at its equilibrium, the betatron wavenumber is given as $k_{\beta}=\varepsilon / a^{2}$, where $\varepsilon$ and $a$ is the beam emittance and equilibrium beam radius. The equilibrium betatron 
wavelength for a $1 \mathrm{~cm}$ radius beam on the $\mathrm{D}-\mathrm{X}$ beamline is about $20 \mathrm{~m}$. Equation (5) becomes

$$
L_{t r}=\frac{6.37 \gamma}{I[\mathrm{kA}]} \sqrt{\frac{\sigma\left[\mathrm{sec}^{-1}\right]}{10^{17} \tau[\mu \mathrm{s}]}}\left(\frac{b[\mathrm{~cm}]}{10}\right)^{3} \frac{\varepsilon[\mathrm{cm}-\mathrm{mr}]}{a^{2}[\mathrm{~cm}]}[\mathrm{m}]
$$

The characteristic growth length $\left(l_{t r}\right)$ in a drift region is given as

$$
\begin{aligned}
l_{t r} & =\sqrt{\frac{\gamma \beta I_{o}}{2 I}} \sqrt[4]{\frac{\pi \sigma}{\tau}} \sqrt{\frac{b^{3}}{c}} \\
& =5 \sqrt{\frac{2 \gamma}{\pi I[\mathrm{kA}]}} \sqrt[4]{\frac{\sigma\left[\mathrm{sec}^{-1}\right]}{10^{17} \tau[\mu \mathrm{s}]}} \sqrt{\frac{b^{3}\left[\mathrm{~cm}^{3}\right]}{1000}}[\mathrm{~m}]
\end{aligned} .
$$

Comparing Eqs. (5) and (7), we observe that $l_{t r}=\left(L_{t r} / k_{\beta}\right)^{1 / 2} / 2$.

According to Eqs. (5) and (7), the instability's growth length increases as the current pulse length decreases. Since the insatbility is less of a problem for a short pulse beam, it is desirable to chop a $2 \mu$ s long pulse to shorter pulses as soon as possible. Equations (5) and (7) also suggest that we can minimize the instability in three ways. First, we can reduce the image current's diffusion rate by using a highly conductive material for the beampipe wall (such as aluminum as in contrast to stainless steel). Second, we can decrease the strength of the magnetic force of the image current by increasing the pipe radius. Finally, we can use relatively strong focusing fields. Two pipe materials are examined for the instability growth: 304 stainless steel with $\sigma=1.25 \times 10^{16}$ $\mathrm{sec}^{-1}$ at $68 \mathrm{~F}^{\mathrm{o}}$, and 6061-TO Al with $\sigma=2.368 \times 10^{17} \mathrm{sec}^{-1}$ at $68 \mathrm{~F}^{\mathrm{o}}$. We let the nominal pipe radius be $8 \mathrm{~cm}$. The $\mathrm{D}-\mathrm{X}$ transport uses a discrete focusing system. To estimate the transverse resistive wall instability's growth rate on the D-X transport, we assume that the system is weakly focusing continuously with an averaged betatron wavelength of 20 $\mathrm{m}$. We calculate the characteristic growth lengths for the drift section and for the overall system for a $2 \mu \mathrm{s}$ long, $2 \mathrm{kA}$ and $20 \mathrm{MeV}$ beam. The results are listed in Table 1.

\begin{tabular}{|c|c|c|c|}
\hline Pipe material & Conductivity & $\begin{array}{c}\text { Overall System's } \\
\text { Growth Length }\end{array}$ & $\begin{array}{c}\text { Growth Length in } \\
\text { Drift Region }\end{array}$ \\
\hline 304 SS & $1.25 \times 10^{16} \mathrm{sec}^{-1}$ & $51.2 \mathrm{~m}$ & $6.4 \mathrm{~m}$ \\
\hline 6061-TO Al & $2.368 \times 10^{17} \mathrm{sec}^{-1}$ & $222.8 \mathrm{~m}$ & $13.3 \mathrm{~m}$ \\
\hline
\end{tabular}

Table 1 Characteristic growth length for the resistive wall instability of a $2 \mu \mathrm{s}$ long, $2 \mathrm{kA}$ and $20 \mathrm{MeV}$ beam on the Diagnostic-X beamline

Table 1 indicates that growth of the transverse resistive wall instability on any one of the D-X beamlines would be reasonable for a full $2 \mu$ s long current pulse if the $8 \mathrm{~cm}$ radius pipe is made out of 6061-TO Al while each individual beamline does not exceed 
$200 \mathrm{~m}$ in length. According to Eq. (7), using a $13 \mathrm{~cm}$ radius stainless steel beampipe would also give the same instability growth length. There is some concern over spilling the beam on the aluminum pipe wall due to its low melting temperature. However, the nominal beam has a radius of $1 \mathrm{~cm}$, which is much less than the pipe radius of $8 \mathrm{~cm}$. The chance of having the beam hitting the wall should be reasonably small. If the beam does hit the wall, its current density would be too small to melt the wall. Using a large stainless steel pipe also has its transport concerns. To minimize the nonlinear fringe fields on the axis, the ratio of a magnet's length to its bore size is usually kept at a constant value. Therefore, the bending systems with larger pipe size would also be linearly proportional to the pipe size. In general, the lengths of the straight sections using discrete focusing systems do not have to increase linearly with the pipe size. However, the geometry constraint of having all beamlines meet at the same firing force them also increase linearly with the pipesize. The entire D-X transport system would then be $60 \%$ longer which is not desirable in terms maintaining the beam quality and the cost. Furthermore, extracting out of the DARHT-II beamline with a limited available space also precludes us from using a large stainless steel pipe. Finally, beam position monitors with a larger radius will be needed if the beampipe is larger. The accuracy of a beam position monitor is typically limited to a few percent of the size of the monitor. Assume that the monitor's radius is $12 \mathrm{~cm}$ for a $13 \mathrm{~cm}$ radius pipe, and the monitor's accuracy is $2.5 \%$. Using this monitor for beam steering would lead to a $\pm 3 \mathrm{~mm}$ beam centroid displacement. An offset beam would propagate down the beamline with betatron oscillations. The total energy of this system is higher than that of a system with the beam perfectly centered. The difference in the total energy, i.e., free energy, can be thermalized by any nonlinear forces within the system. The maximum emittance growth ${ }^{4}$ of the offset beam is given by

$$
\frac{\varepsilon_{f}}{\varepsilon_{i}} \approx\left[1+2\left(\frac{\Delta}{a_{i}}\right)^{2} \frac{k_{\beta}^{2}}{k_{\beta i}^{2}}\right]^{1 / 2},
$$

where $k_{\beta}$ and $k_{\beta i}$ is the betatron wavenumber with and without space charge suppression, respectively, $\varepsilon_{f}$ and $\varepsilon_{i}$ are the final and initial emittance, and $a_{i}$ is the initial beam radius. The nominal beam radius in the $\mathrm{D}-\mathrm{X}$ beamline is $1 \mathrm{~cm}$. The beam emittance can grow by to $10 \%$ over a distance of $120 \mathrm{~m}$ if the beam is offset by $3 \mathrm{~mm}$. Based on all the argument given above, we have determined that the $\mathrm{D}-\mathrm{X}$ beampipe should be made out of aluminum.

Table 1 also indicates that all the individual drift sections on D-X should be much shorter than $10 \mathrm{~m}$. Note that the characteristic growth lengths given in Table 1 are for the full $2 \mu$ s long current pulse, and the beams on most of the D-X beamline would be short pulses ( $100 \mathrm{~ns})$ extending over $2 \mu$ s instead. Therefore, the resulting growth lengths are expected to be longer than that given in Table 1 . The exact growth lengths on all D-X beamlines should be modeled computationally to verify our discussion here.

\section{II.2 Parasitic Energy Loss}


When the beam is traveling down a beampipe, the return current flows in the resistive pipe wall in the opposite direction and creates a longitudinal voltage drop along the wall. The frequency dependent surface impedance of the pipe is given as

$$
Z_{s}(\omega)=\frac{1}{b c} \sqrt{\frac{i \omega}{\pi \sigma}}=\frac{30}{b[\mathrm{~cm}]} \sqrt{\frac{i \omega}{\pi \sigma\left[\mathrm{sec}^{-1}\right]}}[\mathrm{W} / \mathrm{cm}]
$$

The longitudinal electric field corresponding to the ohmic energy loss is

$$
E_{z}(\tau)=\int_{0}^{\infty} \tilde{I}(\omega) Z_{s}(\omega) e^{-i \omega \tau} d \omega / \sqrt{2 \pi}
$$

where

$$
\tilde{I}(\omega)=\int_{0}^{\infty} I(\tau) e^{i \omega \tau} d \tau / \sqrt{2 \pi}
$$

Assume that the beam current has the waveform

$$
I(\tau)=I\left(1-e^{-\tau / \tau_{o}}\right)
$$

with a rise time $\tau_{\mathrm{r}}=\ln 9 \tau_{\mathrm{o}}$. For a $5 \mathrm{~ns}$ rise time, $\tau_{\mathrm{o}}=2.28 \mathrm{~ns}$. After the beam has traveled a distance $\mathrm{L}$, the ohmic energy loss is given by

$$
\Delta W=\frac{I}{I_{o}} \frac{m c^{2}}{2 \pi} \frac{L}{b} \frac{1}{\sqrt{\sigma \tau}}\left[\sqrt{\frac{\tau}{\tau_{o}}}+\frac{2 \pi}{\sqrt{2}} e^{-\tau / \tau_{o}}\right] .
$$

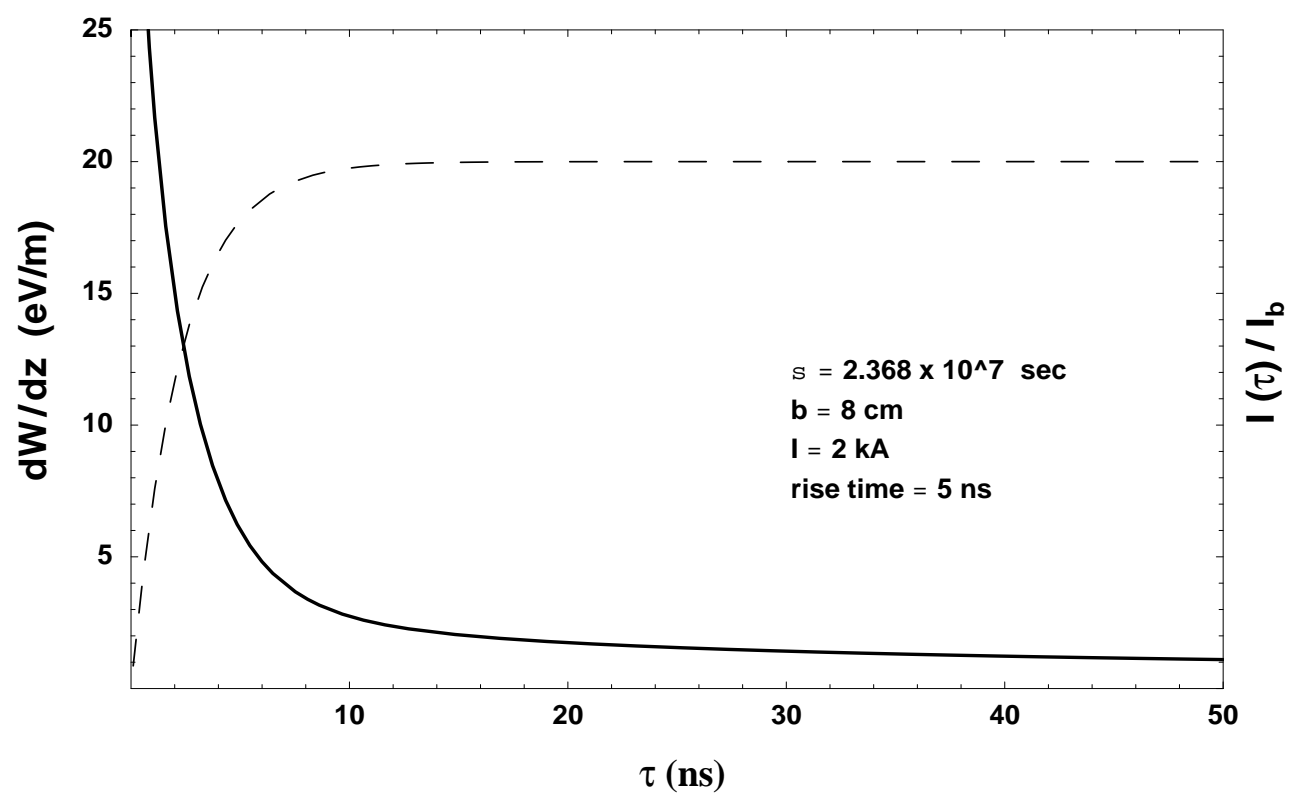

Fig. 2 Parasitic energy loss caused by a resistive wall 
For a $2 \mathrm{kA}$ beam pulse with a $5 \mathrm{~ns}$ rise time traveling for $100 \mathrm{~m}$ in a $8 \mathrm{~cm}$ radius Al pipe, the energy loss per unit length is shown in Fig. 2. The current waveform in arbitrary units is shown in the dashed curve. The energy loss rate where the current is $50 \%$ of the peak current is only about $1 \mathrm{keV}$ which indicates that ohmic loss is not an issue for the Diagnostic $\mathrm{X}$ beam transport.

\section{II.3 Longitudinal Resistive Wall Instability}

The ohmic losses due to the image currents flowing in the resistive wall coupled with perturbations in the current's line density lead to growth of the negative mass instability. This instability is called the longitudinal resistive wall instability. The previous section has already demonstrated that the ohmic losses are quite small for the D$\mathrm{X}$ transport. For a relativistic beam, the relative change in the longitudinal velocity due to the energy loss is even smaller than the relative change in the beam energy, and it is given by

$$
\frac{\Delta \beta}{\beta} \approx \frac{\Delta \gamma}{\gamma} \frac{1}{\gamma^{2}}
$$

The beam needs to travel a reasonably long distance before accumulating enough perturbation in the line density from bunching or debunching. Therefore, for the D-X transport with each beamline length around $100 \mathrm{~m}$, the longitudinal resistive wall instability should not be a concern either. However, we will estimate the instability's growth length here to complete the examination of the wall resistivity effects on beam transport. The longitudinal resistive wall instability depends on the growth of a space charge wave with a phase velocity less than the beam velocity. Hence, the instability can be damped by Landau damping. In other words, growth or damping of the wave depends on the details of the distribution of the particles' longitudinal velocity near the phase velocity. For simplicity, we use the growth length, for a beam without any longitudinal momentum spread ${ }^{4}$, given by

$$
L_{l}=2 \sqrt{\frac{2 \pi \sigma\left[\mathrm{sec}^{-1}\right]}{\omega}} \sqrt{\frac{g \gamma \beta I_{o}}{I}} \frac{b[\mathrm{~cm}]}{100}[\mathrm{~m}]
$$

where $g=1 / 2+2 \ln (b / a)$ is the geometric factor for an emittance dominated beam, and $a$ is the beam radius. We once again let the $\mathrm{D}-\mathrm{X}$ beampipe be a $8 \mathrm{~cm}$ radius Al pipe. For a nominal $2 \mathrm{kA}, 20 \mathrm{MeV}, 1 \mathrm{~cm}$ radius, emittance-dominated beam pulse, the growth length is $98 \mathrm{~km}$ for the longitudinal resistive wall instability at $1 \mathrm{GHz}$ and $219 \mathrm{~km}$ for the instability at $200 \mathrm{MHz}$.

\section{Background Gas Effects and Vacuum Requirement}

A background gas or plasma may affect the beam propagation in a substantial way. Depending on the vacuum pressure, ionizing collisions between electrons and gas molecules may lead to partial or full charge neutralization of the beam. Secondary 
electrons created in these collisions are expelled by the beam electrons' space charge fields, while the ions remain trapped in the potential well of the electron beam. Those ions forming an ion channel can affect electron beam transport by two ways: (1) providing the background gas focusing forces and (2) causing the ion hose instability ${ }^{7-9}$. Finally, the electron beam will be scattered by the background gas molecules which leads to an emittance growth.

\section{III.1 Background Gas Effects}

The resulting charge neutralization factor, $f_{e}(\tau)$, can be approximated as a function of the beam time $\tau$ by

$$
f_{e}(\tau)=\left\{\begin{array}{lll}
\frac{\tau}{\tau_{N}} & \text { for } & 0 \leq \tau \leq \tau_{N} \\
l & \text { for } & \tau>\tau_{N}
\end{array}\right.
$$

where the neutralization time $\tau_{N}$ is approximately $10^{-9} / P$ [torr] for a relativistic electron beam propagating in $\mathrm{N}_{2}$ and about 4 times longer for $\mathrm{H}_{2}$, and $P$ is the vacuum pressure. The r.m.s. beam envelope $R$ is the solution of the envelope equation

$$
R^{\prime \prime}+\frac{(\gamma \beta)^{\prime}}{\gamma \beta} R^{\prime}+k_{\beta}^{2} R-\frac{I}{\gamma^{3} \beta^{3} I_{o} R}\left[1-f_{e}(\tau) \gamma^{2}\right]-\frac{\varepsilon^{2}}{R^{3}}=0,
$$

where $k_{\beta}$ is the betatron wavenumber, and $\varepsilon$ is the unnormalized emittance. Note that compared against the beam's space charge forces, the gas focusing forces of these ions are much stronger by a factor of $\gamma^{2} f_{e}(\tau)$. A large difference in the accumulated phase advance due to the time varying gas focusing effects over a long distance can cause beam envelope oscillations within a pulse, and hence, increase the time integrated spot size. The accumulated difference in the phase advance between the beam head and the beam tail over a distance $\mathrm{L}$ can be approximated as

$$
\Delta \phi \approx \sqrt{\frac{I}{\gamma \beta^{3} I_{o}} \tau_{p}[\mathrm{~ns}] P[\text { torr }]} \frac{L}{R} \quad,
$$

To achieve a small time integrated spot size, the difference in the phase advance should be much less than $2 \pi$. For a $2 \mu \mathrm{s}$ long, $2 \mathrm{kA}$ and $20 \mathrm{MeV}$ beam propagating in a $120 \mathrm{~m}$ long beamline, the pressure needs to be much less than $5 \times 10^{-8}$ torr. However, the electron beam is $2 \mu$ s long only while it is being extracted from the DARHT-II beamline. It then will be chopped into four shorter pulses with the longest pulse being about $500 \mathrm{~ns}$ wide. The subsequent kickers will chop those four pulses further to even shorter pulses. Most of those ions trapped by the electron beam's space charge potential well would escape to the wall quickly during the pulse separation time. Therefore, a low vacuum at $\sim 10^{-8}$ torr should be sufficient. 


\section{III.2 Ion Hose Instability}

Another transport issue concerning the background pressure is the ion-hose instability. If the electron beam is not collinear with a straight ion channel, the electrons tend to follow the displaced ions and perform betatron oscillations around the ion channel with constant amplitude as the beam travels downstream. However, ions in the channel created by the beam are also mobile on the timescale of the beam pulse. Attracted by the oscillating electrons, the ion channel is distorted and forms a sinusoidal channel with a wavelength the same as the electrons' betatron wavelength. The distorted ion channel will then make electron betatron oscillations grow with time and distance. The ion hose instability grows fastest when the beam travels in a preformed offset ion channel with a constant strength, and all the ions oscillate around the displaced electron beam with the same frequency. For a beam with a uniform current distribution, those ions oscillate with a unique frequency $f_{i}$ given by

$$
f_{i} \cong \sqrt{\frac{\pi}{2} \frac{I}{I_{o}} \frac{m}{M}} \frac{c}{a},
$$

where $m / M$ is the mass ratio between electron and ion, $c$ is light speed, and $a$ is the beam radius. For a uniform $2 \mathrm{kA}$ beam with a $1 \mathrm{~cm}$ radius, the oscillation frequency for $\mathrm{H}_{2}{ }^{+}$is $38 \mathrm{MHz}$. However, with a nonuniform electron beam, ions born at different radii will have different oscillation frequencies. A spread in ion's mass or oscillation frequency leads to a smaller "net" ion channel displacement, which damps the instability growth rate $^{8}$. Since ions are trapped by the beam's space charge potential, changing the beam envelope results in changing the ion frequency. In general, the electron beam envelope is seldom constant along a transport line either due to an intentional envelope change such as throwing a beam through a kicker system to a small spot at several meters away, or an un-intentional small envelope mismatch. In either case, the ion hose instability is detuned when the beam envelope changes as it travels from one location to another. Therefore, the instability's growth rate is further reduced ${ }^{9}$. In order to provide reasonable safety margin, we use the instability growth for the worse case with a Bennet electron beam profile to determine the vacuum requirement. The maximum number of e-folding growth $^{10}(\Gamma)$ for such case is given as

$$
\Gamma \cong \frac{3 I f_{e} L}{\gamma \beta^{3} I_{o} k_{\beta} a^{2}},
$$

where $L$ is the distance traveled by the beam. For an emittance dominated beam at its equilibrium, the number of e-folding becomes

$$
\Gamma \cong \frac{3 I f_{e} L}{\gamma \beta^{3} I_{o} \varepsilon},
$$


Let $d$ be the initial separation between the beam and the ion channel, and $\lambda$ be the ion hose instability's transverse beam displacement normalized to the beam radius. We now have $\lambda=(d / a) e^{\Gamma}$. Rearranging Eq. (20) yields to

$$
P[\text { torr }] \cong \frac{\gamma \beta^{3}}{3 \tau_{p}[\mathrm{~ns}]} \frac{I_{o}}{I} \varepsilon[\mathrm{cm}-\mathrm{rad}] \ln \left(\lambda \frac{a}{d}\right)
$$

To obtain a small time integrated $x$-ray spot size without over-focusing the beam, the transverse beam motion at a converter target should not be greater than $10 \%$ of the beam size, i.e., $\lambda \leq 0.1$. According to Eq. (21), for a $2 \mu$ s long, $2 \mathrm{kA}$ and $20 \mathrm{MeV}$ beam with a radius of $1 \mathrm{~cm}$ to propagate on a $\mathrm{D}-\mathrm{X}$ beamline, the pressure should not exceed $1 \times 10^{-8}$ torr if the initial beam-channel separation is $0.5 \mathrm{~mm}$. Note that the pressure requirement is a function of the initial beam-channel separation which is not known at this point. Hence, the current D-X transport system is designed for a vacuum of $1 \times 10^{-8}$, torr with the possibility of lowering the pressure even further if is needed.

The ion hose instability approaches its saturation level when the forces between the beam and the ion channel become nonlinear. This occurs when the separation between the beam and the channel is comparable to or greater than the beam radius, i.e., $|\Delta-\eta| \sim a$, where $\Delta$ and $\eta$ are the transverse displacement for the channel and the beam, respectively. The equation of motion governing the channel's coherent transverse displacement is

$$
\frac{\partial^{2} \Delta}{\partial t^{2}}+\omega_{i}^{2} \Delta=\omega_{i}^{2} \eta
$$

Let $\Delta$ and $\eta$ vary as $\exp (-i \omega \tau)$, where $\omega$ can be complex. The ion channel is an undamped harmonic oscillator as shown by Eq. (22). When it is driven at its resonance frequency, its amplitude is much larger than the driver's amplitude, i.e.,

$$
\frac{\eta}{\Delta} \sim \frac{\omega_{i}^{2}-\omega^{2}}{\omega_{i}^{2}} \sim \frac{2 \delta \omega}{\omega_{i}}<<1,
$$

where $\delta \omega$ is either the frequency spread or the growth rate. Hence, when the instability becomes nonlinear, the magnitude of the ion channel's transverse displacement approaches the beam radius, and the electron beam's displacement stays much smaller than the beam size. This indicates that the ion hose instability's amplitude should always be small compared with the beam radius. These results have been observed in ion hose instability simulations ${ }^{11}$. Therefore, although the spatial and temporal growth of the ion hose instability for small displacements is generally rapid for parameters of practical interest, the instability may not be as threatening as indicated by the linear growth theory. This may lead to relaxing the vacuum requirement in the system. Nevertheless, experiments are needed to validate this argument. 


\section{III.3 Background Gas Scattering}

Scattering in a background gas can cause emittance growth. The growth rate for the normalized emittance is given as

$$
\frac{d \varepsilon_{n}}{d z} \cong \frac{7}{k_{\beta}} P[\text { torr }] \frac{Z_{g}^{2}}{\gamma \beta^{3}} \ln \left(\frac{204}{Z_{g}}\right)[\mathrm{mm}-\mathrm{mrad} / \mathrm{m}]
$$

where $Z_{g}$ is the charge of the gas atom. Let us assume that $\lambda_{\beta}$ is $20 \mathrm{~m}$ again. For a 20 $\mathrm{MeV}$ beam traveling $120 \mathrm{~m}$ in $10^{-8}$ torr of oxygen, the total emittance growth caused by background gas scattering is $1.38 \times 10^{-3} \pi \mathrm{mm}-\mathrm{mr}$ which is insignificant compared with the nominal $1000 \pi \mathrm{mm}$-mr emittance exiting the DARHT-II accelerator.

\section{Emittance Growth in Bends}

To bring four beamlines to the same firing point and to extract the beam out of the DARHT-II beamline requires multiple bends. On a typical D-X beamline, the beam has to travel through 4 bending systems (one line has 5 bends) and two active kickers. The DARHT-II emittance specifications are $1000 \pi \mathrm{mm}-\mathrm{mr}$ at the accelerator exit, and an allowance for the emittance to grow by $50 \%$ to $1500 \pi \mathrm{mm}-\mathrm{mr}$ at the target. In Sec. I, we have established the maximum acceptable emittance at the D-X targets as $1875 \pi \mathrm{mm}-\mathrm{mr}$ (an $87.5 \%$ increase). Based on this $87 \%$ growth budget and what we have learned from modeling the DARHT-II kicker system with simulations, a $10 \%$ emittance growth is allocated for each D-X kicker. If the goal is to meet the $1500 \pi \mathrm{mm}-\mathrm{mr}$ design specification, and if the emittance budget for each bending system is $5 \%$, there will be only a $10 \%$ emittance growth budget for beam transport in the lengthy straight sections of the beamline and at the beam-target interaction point. If the goal is to satisfy the 1875 $\pi \mathrm{mm}$-mr emittance goal, the emittance budget for the straight sections and the beamtarget interaction would be $47 \%$ which is still tight but reasonable. The simulations of a $3 \mathrm{kA}, 40 \mathrm{MeV}, 1500 \pi \mathrm{mm}-\mathrm{mr}$ beam interacting with a $3 \mathrm{~cm}$ long target plasma channel predicted a $20 \%$ emittance increase for a uniform beam and almost a factor of 2 increase for a Gaussian beam ${ }^{12}$. The fourth pulse of the four pulse train also has to encounter a long target plasma channel $(\sim 1.6 \mathrm{~cm})$. Simulations are needed to model the emittance growth at the target. Although the plasma channel for the fourth pulse is only half of what was modeled in Ref. 12, the focusing strength is now stronger than that in Ref. 12. We expect a similar emittance growth for this pulse. It is obvious that performance of the D$\mathrm{X}$ depends on whether beam quality can be preserved in all the bends. The relative emittance growth for each bending system should be limited to be much less than $5 \%$.

There are two sources of emittance growth in a bend. The first source is the curvature of the beam and the pipe. There are nonlinear fields in the bend even if both the beam and the pipe have round cross-sections, and the beam density is uniformly distributed. The second source, which exists in the straight beamline also, is the difference in the shape and the orientation of the beam cross-section and the pipe crosssection. Even if the beam density were uniformly distributed, the image charges and the image current would not be uniformly distributed on the pipe wall when the cross- 
sections of the beam and the pipe are different in shape and in orientation. Then, the beam would experience some nonlinear image forces which lead to emittance growth.

To obtain a scaling law for the emittance growth arising from the curvature of a gentle bend such that the beampipe radius is much smaller than the radius of curvature, we ignore the effects of charge redistribution and betatron motion of particles and assume that the nonlinear forces on the beam only add kicks to the particles' transverse velocities. We further assume that the changes in their transverse positions due to these kicks are negligible. For a round, uniform DC beam, the emittance growth ${ }^{13}$ arising from the curvature of the beam and the pipe is obtained as

$$
\begin{aligned}
\Delta \varepsilon_{n, x} & =\Delta \varepsilon_{n, y} \\
& =4 \gamma \beta x_{r m s} \Delta x_{r m s}^{\prime} \\
& \cong \frac{\sqrt{2}}{8} \frac{I}{\beta_{\varphi} I_{o}} \frac{a}{R} a \alpha_{b}+O\left(\left(\frac{a}{R}\right)^{3},\left(\frac{\Delta}{a}\right)^{2}\right) .
\end{aligned}
$$

This beam emittance growth is added in quadrature to the initial beam emittance. In Eq. (24), $\alpha_{b}$ is the bend angle, and $\Delta$ is the transverse beam displacement, which is assumed to be much smaller than the beam radius. Although there is a nonlinear force component to the first order in $\Delta a$, the emittance growth caused by the beam displacement does not appear in first order in $\Delta a$.

In many cases such as the $\mathrm{D}-\mathrm{X}$ beamline, the lengths of the bending magnets on a given beam line are the same. The emittance growth for each bend with a bending magnet length $\ell$ is proportional to square of the bend angle as given by

$$
\Delta \varepsilon_{n, x} \cong \frac{\sqrt{2}}{8} \frac{I}{\beta_{\varphi} I_{o}} \frac{a^{2}}{\ell} \alpha_{b}^{2} .
$$

Assume that we need to bend the beam $N$ times to reach a total bend angle $\alpha_{b, t o t}=N \alpha_{b}$. The total emittance growth is then given as

$$
\Delta \varepsilon_{n, x} \cong \frac{\sqrt{2}}{8 N^{3 / 2}} \frac{I}{\beta_{\varphi} I_{o}} \frac{a^{2}}{\ell} \alpha_{b, t o t}^{2}
$$

It is obvious that bending beams gently is more desirable in terms of emittance preservation if we can afford the lab space for a longer beam line. Let us consider a nominal $\mathrm{D}-\mathrm{X}$ beam, i.e., a $20 \mathrm{MeV}, 2 \mathrm{kA}, 1 \mathrm{~cm}$ radius beam making a $360^{\circ}$ turn by traveling through sixteen $22.5^{\circ}$ bends. Each bend is $50 \mathrm{~cm}$ long ${ }^{1}$. The normalized emittance growth is about $0.64 \mathrm{~mm}$-mrad for one bend, and the total normalized emittance growth is $2.6 \mathrm{~mm}$-mrad. Therefore, the emittance growth from the nonlinear forces arising from the curved geometry is insignificant for the D-X beam transport. 
Most of the emittance growth in a bend would come from the nonuniform image charge and current distributions due to the geometric difference between the crosssections of the beam and the pipe. Typically, a dipole magnet is used to bend the beam. The edge focusing effects of the dipole magnet would focus the beam in the bend plane. However, the beam is simply drifting in the plane perpendicular to the bend plane. Differences in the focusing in these two planes result in a beam with an elliptical crosssection. Unless the beampipe aperture is also elliptical with the same eccentricity and orientation, there would be nonlinear image forces. The typical chicane used by the rf accelerator community has an elongated cavity with the dimension in the non-bend plane longer than that in the bend plane so that the nonlinear image forces can be minimized. This approach is reasonable for the rf machines since a chicane only consists of a few bending magnets. The rf beam size is small, and so is the chicane cavity. Making a small, elongated cavity is not too difficult. However, a typical bending system on the D-X is about $10 \mathrm{~m}$ in length. With many bending magnets and quadrupoles in the system, the shape of the beam cross-section varies from magnet to magnet which makes having the pipe cross-section similar to the beam cross-section extremely difficult and costly. For practical reasons, the pipe cross-section would be round through out the D-X beamline except where the split beampipe is. The $\mathrm{D}-\mathrm{X}$ transport tune should be chosen to make the beam as round as possible and its size much smaller than the pipe size so that the nonlinear image force effects are weaker. Simulations of the emittance growth from the nonlinear image forces in all the D-X bends are needed to verify that the beam quality is preserved in the bends.

A single bending magnetic is dispersive as shown in Fig. 3(a), i.e., the exiting beam orbit varies as a function of the beam energy. Since there will be energy variations within a DARHT-II pulse and between pulses, all the bends need to be achromatic in order to minimize the transverse beam motion (in other words the bends need to be energy insensitive to some approximation). There are several doubly achromatic (to first order) bend configurations possible. The simplest achromatic bend system consists of two bends separated by a quadrupole as shown in Fig. 3(b). If the total bend angle of the system is $\theta$, each bend would bend the beam by $\theta / 2$. Besides its simplicity, the advantage of this bend system is that is has the shortest beamline. However, to bend the required angles on the D-X beamline, this simple system produces a highly elliptical beam (see Fig. 4) leading to an unacceptable emittance growth due to nonlinear image forces. The second system considered consists of four modules. For bend systems without a kicker, each module consists of a focusing quadrupole, a dipole providing a bend $(\theta / 4)$ and a defocusing quadrupole as shown in Fig. 3(c). The focusing and defocusing quadrupole pair prevents the beam from being highly elliptical (see Fig. 5). The emittance growth associated with a less elliptical beam in a round pipe will be more reasonable for the tight $\mathrm{D}$-X emittance budget. The $\theta / 4$ bend angle increases the radius of curvature of the bend, and hence, reduces the nonlinear image fields of the return current. For bend system with a kicker, each module consists of a $\theta / 4$ bend, and a doublet (defocusing \& focusing quadrupole pair). The $\mathrm{D}-\mathrm{X}$ transport will use the 4-module bending system. The simulation results ${ }^{2}$ for these two simplest achromatic bending systems support our choice of the 4-module bending system. We need to ensure that these systems preserve beam quality in the presence of space charge and image forces (these forces are usually 
negligible in high energy physics machines which have very low beam currents and are small but not ignorable in ours).

(a) Dispersive bending magnet

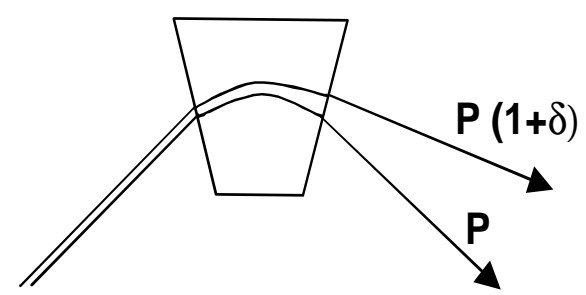

(b) 3-component achromatic bending system
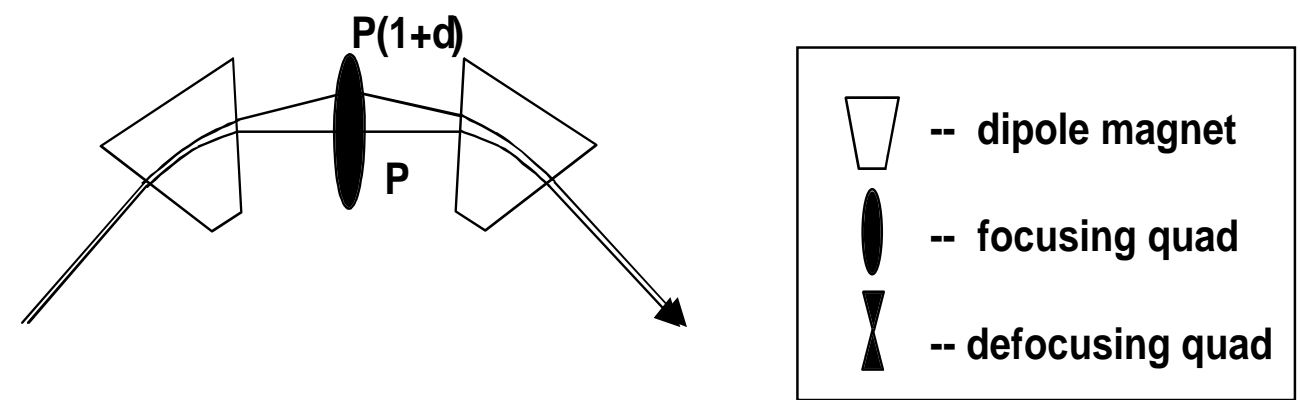

(c) 4-module achromatic bending system

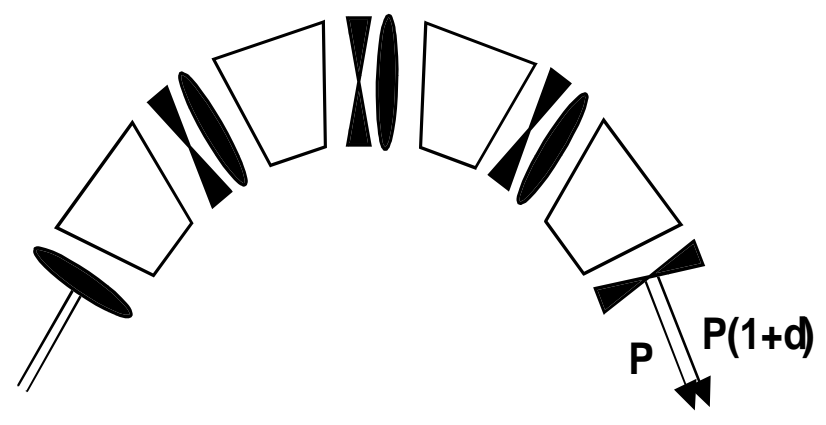

Fig. 3 Three bending system configurations. 

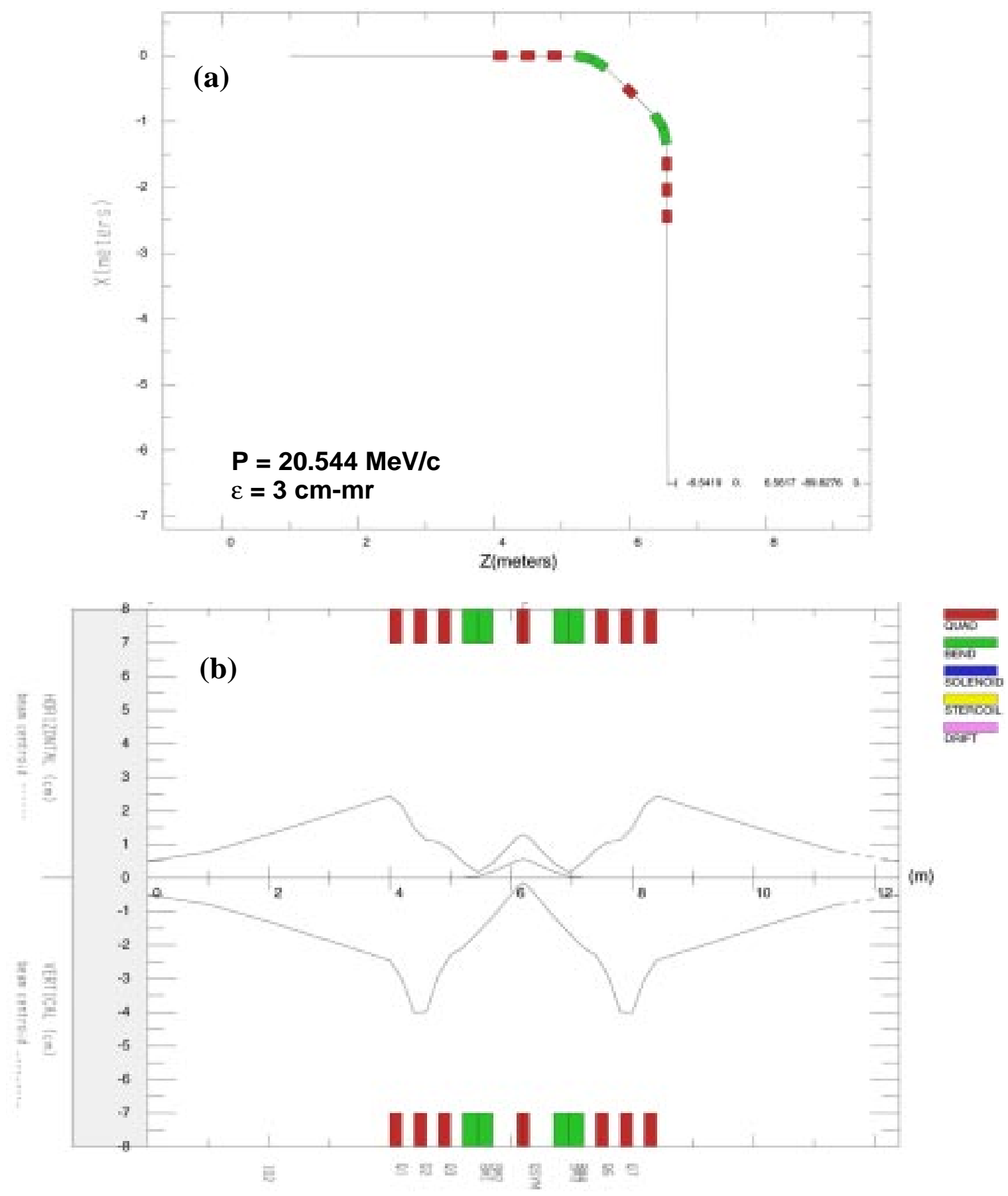

Fig. 4 A 3-element $90^{\circ}$ achromatic bending system for a $20 \mathrm{MeV}$ beam with a $1200 \pi$ $\mathrm{mm}-\mathrm{mr}$ normalized Lapostolle emittance. The beamline layout is shown in (a). The beam envelopes in the bend plane (the upper curve) and in the non-bend plane (the lower curve) are shown in (b). The beam centroid displacement for the beam with an energy different from the design energy by $1 \%$ is also shown in (b). 

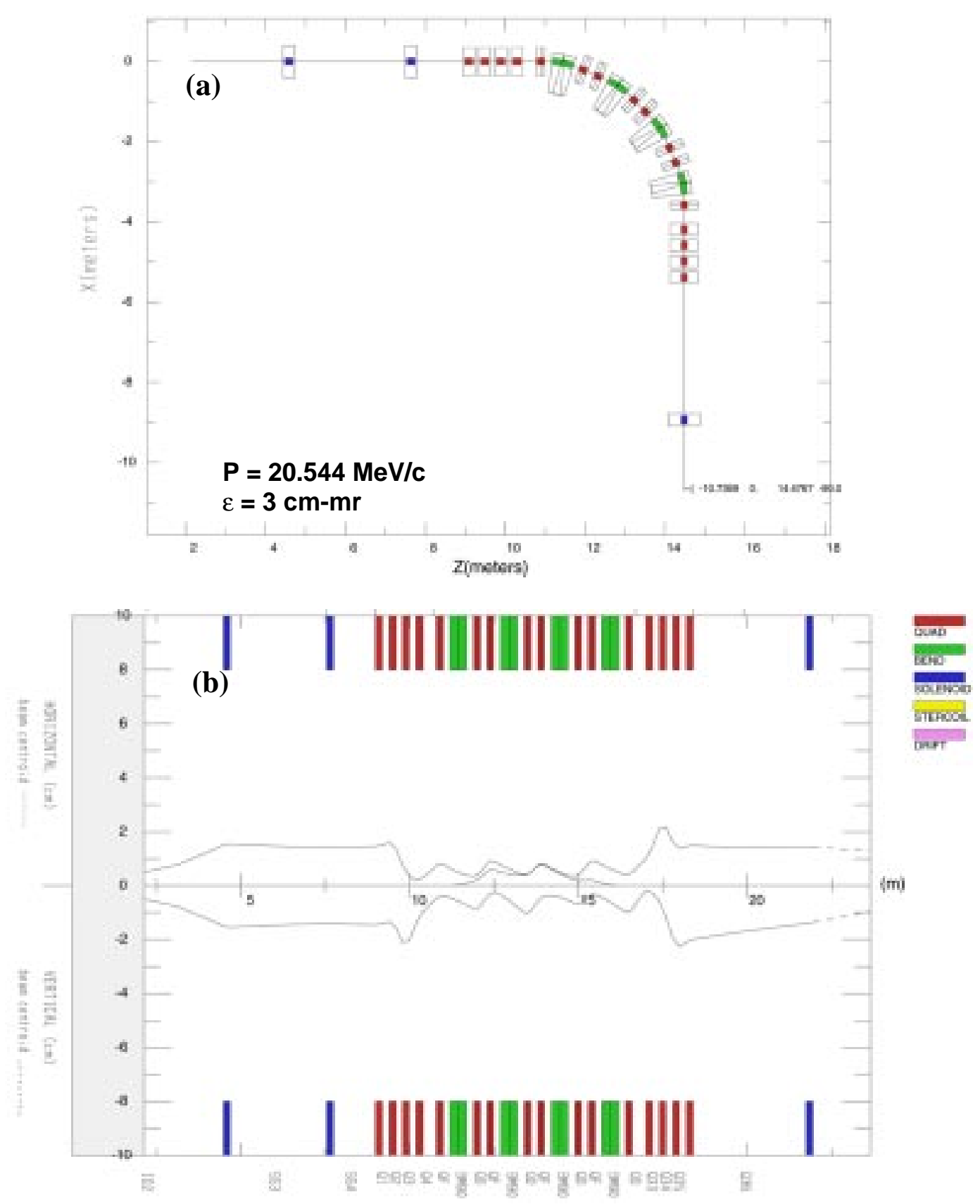

Fig. 5 A 4-module $90^{\circ}$ achromatic bending system for a $20 \mathrm{MeV}$ beam with a $1200 \pi$ $\mathrm{mm}-\mathrm{mr}$ normalized Lapostolle emittance. The beamline layout is shown in (a). The beam envelopes in the bend plane (the upper curve) and in the non-bend plane (the lower curve) are shown in (b). The beam centroid displacement for the beam with an energy different from the design energy by $1 \%$ is also shown in (b). 


\section{Misalignment and Corkscrew Beam Motion}

A large time varying transverse beam motion would lead to a large timeintegrated spot size on the target. A leading cause for the time varying transverse motion is the coupling of chromatic aberration of a misaligned transport system and the time dependent energy variation within a current pulse. When a beam with an energy variation over its flattop propagates in a misaligned transport system, there will be a differential betatron oscillation of the beam centroid between any two slices of the beam. If the phase advance of this differential betatron oscillation were larger than $2 \pi$, the current pulse resembles a corkscrew. Hence, the differential oscillation is called corkscrew motion ${ }^{14,15}$.

Let us consider a transport system consisting of $n$ randomly misaligned magnets. Let $\delta B_{j}$ be the dipole field arising from misalignment of the $\mathrm{j}$-th magnet. Since the electron beam centroid is an undamped harmonic oscillator oscillating at its betatron wavenumber $k_{c}(\tau)$, its oscillation will be excited by the Fourier component of the dipole field at the wavenumber $k_{c}(\tau)$. The corresponding gyro-radius of a given beam slice at time $\tau$ is given by

$$
\rho_{j}(\tau) \approx \int_{-\infty}^{\infty} e^{-i k_{c}(\tau)} \frac{\delta B_{j}}{B_{z}} d z
$$

When the difference in the total phase advance $\left(\phi_{t o t}\right)$ of the beam transport through the entire system is small compared with 1 , the corkscrew amplitude for this transport system with randomly misaligned magnets is given as

$$
\begin{aligned}
|\eta| & \approx n^{3 / 2}\left|\rho_{r m s}\right|\left(\frac{\Delta \gamma}{\gamma}\right) \phi \\
& \approx \sqrt{n}\left|\rho_{r m s}\right|\left(\frac{\Delta \gamma}{\gamma}\right) \phi_{t o t},
\end{aligned}
$$

where $\phi$ is the phase advance after traveling through a single misaligned magnet. The quantity $\rho_{r m s}$ is the r.m.s. value of the electron gyro-radii of the reference beam slice resulting from $n$ randomly misaligned magnets.

We will now try to establish the magnetic alignment specifications for the D-X system. Assume $\left|\rho_{r m s}\right| \sim \Delta \theta_{r m s} l \sim \Delta_{r m s}$, where $\Delta \theta_{r m s}$ is the r.m.s. value of the magnet tilts, $\Delta_{r m s}$ is the r.m.s. value of the magnet offsets, and $l$ is the magnet length (nominally 20 $\mathrm{cm}^{1}$ ). The requirement for the transverse beam motion on the DARHT-II target is that its amplitude should be less than $10 \%$ of the beam radius. The beam typically has to travel through approximately 100 more magnets to reach its D-X converter target than to reach the DARHT-II target. Let us assume that the corkscrew amplitude arising from misalignment of those 100 magnets alone is also one tenth of the beam radius. The corkscrew amplitudes caused by random tilts and offsets generally add in quadrature. The 
net corkscrew amplitude, including the contribution from the misaligned DARHT-II magnets, at a D-X target will be about $14 \%$ of the beam radius. For the worst case, the time integrated D-X spot size is now $14 \%$ larger versus $10 \%$ larger for the time integrated DARHT-II spot size if the same focusing field is used for both systems. We can easily compensate for this difference by increasing the final focusing field at the D-X target area by $4 \%$. The energy variation within the pulse is less than or equal to $\pm 0.5 \%$. The total phase advance on a D-X beamline is nominally 7 × $2 \pi$. We assume that the random tilts and the random offsets contribute to the corkscrew motion equally. According to Eq. (28), the r.m.s. tilt has to be less than $0.8 \mathrm{mrad}$, and the r.m.s. offset has to be less than $0.16 \mathrm{~mm}$ if no dynamic steering would be implemented to control the corkscrew motion. These specifications are very similar to the DARHT-II alignment specifications: the $3 \sigma$ for the random tilts being $1.95 \mathrm{mrad}$ and the $3 \sigma$ for the random offsets being $0.45 \mathrm{~mm}$. Our experience on controlling the corkscrew motion on the ETA$\mathrm{II}^{16}$ and the $\mathrm{FXR}^{16}$ dynamically and the simulations of corkscrew control on the DARHTII indicate that it is reasonable to assume that using the dynamic corkscrew tuning curve algorithm ${ }^{16}$ can reduce the D-X corkscrew amplitude by one order of magnitude. Hence, the corkscrew motion in the D-X transport system is not a concern.

\section{Beam Extraction}

The first design task in the Diagnostic $\mathrm{X}$ beamline is the extraction of the beam from DARHT-2. Extraction of the DARHT-II beam can be accomplished by utilizing the existing DARHT-II transport components and with minimal modification of the DARHTII building. It is also important to allow the beam extraction line to coexist with the DARHT-II shuttle dump so that it can be used to characterize the beam and to tune the DARHT-II accelerator before bending it into the D-X transport system.

The first apparent option is to utilize the DARHT-II kicker system to chop a $2 \mu \mathrm{s}$ long DARHT-II beam into a series of short pulses with the D-X pulse format and to dump the unwanted electrons in the DARHT-II main dump. DARHT-II pulses would then be extracted after the DARHT-II kicker exit, and no modification to the DARHT-II building would be needed. The extracted beam would now be a series of short pulses over a $2 \mu$ s duration. The transverse resistive wall instability due to diffusion of the return current in the beampipe wall is less of a problem for such a beam pulse train as compared with a $2 \mu$ s long pulse. Therefore, by extracting beams after the DARHT-II kicker system, we could use a smaller beampipe for the DX extraction beamline, and hence, magnets with smaller bores, resulting in a bend with a smaller radius of curvature. This would finally lead to an extraction bend with a shorter total beamline length. However, this option was ruled out after the DARHT-II project allocated $4 \mathrm{~m}$ of physical space to clean up the several hundred nanoseconds of beam head. Without this 4 meters, parts of the extraction beamline would be too close to the DARHT-II firing point.

Since we now have to extract the beam upstream of the DARHT-II kicker, the beam will be $2 \mu$ s long with an unknown amount of beam head current. As discussed in Sec. II.1, the D-X beampipe would be made out of a highly conductive material, 6061TO Al, so that the diffusion rate of the wall image current would be reduced in order to 
suppress growth of the transverse resistive wall instability. It is relatively easy to drill a hole through the aluminum pipe of the beam extraction (a $90^{\circ}$ bend) due to its low melting temperature. It is important to characterize the beam parameters well before extracting the beam. The best option is to extract the beam without removing any parts of DARHT-II beamline, especially the beamline leading to the DARHT-II shuttle dump. Putting the beam extraction point in the drift space $(\sim 3 \mathrm{~m})$ upstream of the DARHT-II shuttle dump would allow us to use the shuttle dump for tuning the accelerator and characterizing the beam quality by turning off the first bend on the extraction line.

Once the beam is extracted from the DARHT-II beamline, it needs to exit the DARHT-II building and to be transported to the D-X firing point. Layout of the D-X beamline leaving the building should not weaken the building structure and interfere with the normal DARHT-II operations outside the building. These considerations lead to the choice of extracting the beam out of the DARHT-II beamline and building with a $10^{\circ}$ downward slope and to bend the beam back to the horizontal level under the ground. This additional $10^{\circ}$ bend has led to an approximately $12 \mathrm{~m}$ increase in beamline length.

\section{Modularity of the beamline}

In order to minimize the number of shots needed for tuning the $\mathrm{D}$-X beamline, we are designing the beamline to consist of several "identical" modules, such as $10^{\circ}, 45^{\circ}$ and $90^{\circ}$ achromatic bend systems, $90^{\circ}$ kicker achromatic bend systems, etc. A matching section precedes each module. Operationally, we would tune each type of module once, and then set all the identical module's magnets at the same tune. We then would only tune individual matching sections to match beams into the following modules.

\section{Integrated Spot Size and Final Focus}

The time integrated beam spot size on a converter target is determined by the intrinsic thermal spread of the beam, the spherical and chromatic aberrations of the final focus lens as shown by

$$
a_{f}^{2} \approx\left(\frac{\varepsilon f}{a_{o}}\right)^{2}+\left(C_{s} a_{o}^{3}\right)^{2}+\left(2 \frac{\Delta \gamma}{\gamma} a_{o}\right)^{2},
$$

where $a_{f}$ and $a_{o}$ is the beam radius on the target and entering the final focus lens, respectively, $C_{s}$ is the final focus lens' spherical aberration coefficient. For a beam with a $\pm 0.5 \%$ energy variation, the spot size increase due to chromatic aberration is small if the entering beam radius is $3 \mathrm{~cm}$ or less. The optimal final focusing system usually requires that the contribution from the thermal spread to be the same as that from spherical aberration. To achieve the DARHT-II spot size ( $2.1 \mathrm{~mm}$ diameter), each of the first two terms of the right-hand side of Eq. (29) has to be about $0.7 \mathrm{~mm}$.

The final focus will be that developed for the DARHT-II if the beam emittance is $1500 \pi \mathrm{mm}$-mr or less. The required field strength for the final focusing lens varies from 
$17 \mathrm{kG}$ to $6 \mathrm{kG}$ when the entering beam radius varies from $1.5 \mathrm{~cm}$ to $3 \mathrm{~cm}$. For the magnetic field lower than $10 \mathrm{kG}$, a DC magnet would be used. On the other hand, a pulsed magnet would be needed to provide the final focusing field higher than $10 \mathrm{kG}$. If the final beam emittance were $1875 \pi \mathrm{mm}-\mathrm{mr}$ (see Sec. I), the final focusing field strength needs to be $25 \%$ higher than that for the DARHT-II. It is more likely that the D$\mathrm{X}$ final focus lens may need to be a pulsed magnet.

\section{Conclusions}

Many transport concerns have been discussed in this report. Based on our current knowledge of those potentially degrading mechanisms, we believe that those degrading mechanisms can be suppressed. Although we think that there is no great concern in terms of preserving the beam quality, there are still large uncertainties existing in our knowledge. We have developed a scaling law for the forward x-ray dose as a function of the beam emittance. We understand how the current distribution on the target changes the x-ray spot size corresponding to $50 \%$ of the modulation transfer function. However, how the forward dose changes with the beam distribution on the target is unclear. The transverse resistive wall instability for a long pulse is well understood. However, we need to develop a model for a train of short pulses. The basic mechanism of the ion hose instability is well understood also. However, we lack of the knowledge on the ion distributions and species types, and hence, the exact growth rate and saturation level. There is also questions of how to tune a large angle achromatic bending system without getting a highly elliptical beam since we cannot monitor the beam size inside a magnet with the existing diagnostic tools. There are also design concerns for the beam transport in a kicker system which is discussed in Refs. 2 and 17. And last but not least, the biggest concern in terms of delivering the DARHT-II-like x-ray pulse format is the beam-target interaction. The D-X converter target will be that developed for the DARHT-II. Even if the target works for the DARHT-II, it still needs to be verified for the D-X beam parameters. All of these uncertainties mentioned above need to be tested on the ETA-II so that our theoretical understanding can be verified, our computational tools can be benchmarked, and ultimately the $\mathrm{D}-\mathrm{X}$ beam transport can meet its performance specifications.

\section{Acknowledgements}

This work was performed under the auspices of the U.S. Department of Energy by the University of California Lawrence Livermore National Laboratory under contract No. W-7405-Eng-48.

\section{References}

1. M. Burns, et. al., "DARHT Accelerator Update and Plans for Initial Operation", Proc. of 1999 Particle Accelerator Conference, New York, N. Y. March 27 - April 2, 1999, p. 617.

2. B. R. Poole, "Emittance Growth in Diagnostic X Transport Line", (this D-X closeout document). 
3. A. C. Paul, "Diagnostic X Multi-Axis Beamline", (this D-X closeout document), April, 2000.

4. Y.-J. Chen and E. A. Rose, "The Effects of Beam Emittance and Final Spot Size on the Forward X-Ray Dose", memo, April 2000.

5. G. J. Caporaso, W. A. Barletta, and V. K. Neil, Particle Accelerator, 11, 71, (1980).

6. M. Reiser, "Theory and Design of Charge Particle Beams", John Wiley\& Sons (1994).

7. H. L. Buchanan, "Electron Beam Propagation in the Ion-Focused regime", Phys. Fluid, 30, 221, (1987).

8. G. V. Stupakow, "Comment on "Natural BNS Damping of the Fast Ion Instability", Phys. Rev. Special Topics-Accel. And Beams, 3, 19401 (2000).

9. G. J. Caporaso and J. F. McCarrick, "Ion-Hose in DARHT-2", Proc. Of the DARHTII Ion Hose Workshop, LBNL, Feb. 8, 2000.

10. R. Briggs, "Transverse Instabilities From Ion Oscillations in the DARHT-II Accelerator", LBNL Engineering Note \#M7848, Jan. 25, 2000.

11. T. C. Genoni and T. P. Hughes, "Ion-Hose Calculations", Proc. Of the DARHT-II Ion Hose Workshop, LBNL, Feb. 8, 2000.

12. B. Oliver, et. al, "Beam-Target Interactions in Single- and Multi-Pulse Radiography”, Mission Research Report No. MRC/ABQ-R-1909, April 1999.

13. Y.-J. Chen, "Space-Charge Forces of a DC Beam in a Continuous Bend", Proc. 1997 Particle Accelerator Conference, Vancouver, B. C. Canada, May 12-16, 1997, p. 1864.

14. Y.-J. Chen, "Corkscrew Modes in Linear Accelerator", Nucl. Instr. and Meth. A292, 455 (1990).

15. Y.-J. Chen, "Control of Transverse Motion Caused by Chromatic aberration and Misalignments in Linear Accelerators", Nucl. Instr. and Meth. A 398, 139 (1997).

16. R. Scarpetti, Private communications.

17. L. Wang and B. R. Poole, "Dipole Septum Magnet in the Fast Kicker System for Diagnostic X", (this D-X closeout document). 